\title{
Livistona chinensis seed suppresses hepatocellular carcinoma growth through promotion of mitochondrial-dependent apoptosis
}

\author{
WEI LIN ${ }^{1,2}$, JINYAN ZHAO $^{1,2}$, ZHIYUN CAO $^{1}$, QUNCHUAN ZHUANG $^{1,2}$, LIANGPU ZHENG $^{1,2}$, \\ QIAOYAN CAI ${ }^{1,2}$, DAXIN CHEN ${ }^{1,2}$, LILI WANG ${ }^{1,2}$, ZHENFENG HONG ${ }^{1}$ and JUN PENG ${ }^{1,2}$ \\ ${ }^{1}$ Academy of Integrative Medicine; ${ }^{2}$ Fujian Key Laboratory of Integrative Medicine on Geriatrics, \\ Fujian University of Traditional Chinese Medicine, Fuzhou, Fujian 350122, P.R. China
}

Received November 14, 2012; Accepted February 4, 2013

DOI: $10.3892 /$ or.2013.2319

\begin{abstract}
The Livistona chinensis seed has been used for centuries to clinically treat various types of cancer. However, the precise mechanism of its anticancer activity remains to be elucidated. In the present study, we evaluated the efficacy of the ethanol extract of Livistona chinensis seed (EELC) against tumor growth using a hepatocellular carcinoma (HCC) mouse xenograft model and an HCC cell line, HepG2, and investigated the molecular mechanisms mediating its biological activities. We found that EELC inhibited HCC growth both in vivo and in vitro, without apparent signs of toxicity. In addition, EELC treatment resulted in the induction of HCC cell apoptosis. Moreover, EELC-induced apoptosis was accompanied by the loss of mitochondrial membrane potential, activation of caspase- 9 and caspase-3, and increase in the proapoptotic $\mathrm{Bax} / \mathrm{Bcl}-2$ ratio. Our findings suggest that promotion of mitochondrial-dependent apoptosis in cancer cells may be one of the mechanisms by which the Livistona chinensis seed is effective in cancer treatment.
\end{abstract}

\section{Introduction}

Hepatocellular carcinoma (HCC) is one of the most common types of cancer worldwide, with nearly one million new cases and over 600,000 deaths annually (1-5). Although surgical resection offers the best prognosis for long-term survival, the majority of HCC patients are not eligible for surgery as at the

Correspondence to: Professor Zhenfeng Hong or Dr Jun Peng, Academy of Integrative Medicine, Fujian University of Traditional Chinese Medicine, 1 Huatuo Road, Minhou Shangjie, Fuzhou, Fujian 350122, P.R. China

E-mail: zfhong1953@163.com

E-mail: pjunlab@hotmail.com

Abbreviations: HCC, hepatocellular carcinoma; EELC, ethanol extract of the seeds of Livistona chinensis; DMSO, dimethyl sulfoxide; MTT, 3-(4,5-dimethylthiazol-2-yl)-2,5-diphenyltetrazolium bromide

Key words: hepatocellular carcinoma, apoptosis, herbal medicine, mitochondrion, Livistona chinensis seed time of diagnosis the tumor may be too large, or it may have expanded into nearby major blood vessels or metastasized (6). Therefore, chemotherapy remains a major therapeutic approach for patients with advanced HCC. Regimens including doxorubicin, cisplatin or fluorouracil, as single agents or in combination, are the standard treatments for these patients. However, due to drug resistance, systemic chemotherapy using the above regimens produces a disappointing low response rate (7). Moreover, several currently used anticancer agents have potent cytotoxic effects in normal cells (8). These problems limit the effectiveness of current HCC chemotherapy, increasing the necessity for the development of novel anticancer agents. Natural products have received attention as they have relatively few side-effects and have long been used as alternative remedies for a variety of diseases including cancer $(9,10)$. Thus, identifying naturally occurring agents is a promising approach for anticancer treatment.

The biological role of apoptosis is to eliminate redundant or damaged cells and, hence, is crucial for maintaining tissue homeostasis. Disturbed regulation of this vital physiological process underlies numerous diseases including cancer (11-13). Apoptosis can be triggered by either intrinsic stimuli, such as cytokine deprivation and DNA damage, or by extrinsic stimuli, such as death ligand-receptor engagement. Both intrinsic and extrinsic signals eventually lead to the activation of cysteine-dependent aspartate-directed proteases (caspases) and nucleases, resulting in destruction of the cell $(13,14)$. The best understood intrinsic apoptotic pathway is centered at the mitochondria, which is therefore referred to as mitochondrialdependent apoptosis. Bcl-2 family proteins are key regulators of mitochondrial-dependent apoptosis $(12,13)$, functioning as either suppressors, such as Bcl-2, or promoters, such as Bax. One mechanism by which Bcl-2 family proteins regulate apoptosis is through their effect on the permeability of the mitochondrial outer membrane (MOM) via homo- or hetero-association (15). Activation of either of the pro-apoptotic proteins Bax or Bak is sufficient to induce mitochondrial outer membrane permeabilization (MOMP) (16-19). This event leads to the release of pro-apoptotic proteins such as cytochrome $c$ and Diablo/Smac that, in turn, trigger the activation of the caspase cascade (19-23). The anti-apoptotic $\mathrm{Bcl}-2$ protein protects cells from apoptosis by interacting with Bax and inhibiting Bax-mediated MOMP $(16,23,24)$. The ratio of active anti- and 
pro-apoptotic Bcl-2 family proteins determines the fate of cells. Alteration of this ratio by aberrant expression of these proteins impairs the normal cellular apoptotic program and may contribute to various apoptosis-related diseases including several types of cancer $(25,26)$. Therefore, promoting cell apoptosis via regulation of the Bcl-2 family proteins has been a major focus in the development of anticancer therapies.

Livistona chinensis, belonging to the monocotyledonous Palmaceae family, is a medicinal herb widely distributed in Eastern Asia. The seed of Livistona chinensis has long been used in China to clinically treat various types of cancer (27). Extracts of the Livistona chinensis seed have been shown to inhibit the growth of several cancer cells (28-31). However, the precise mechanisms of its tumoricidal activity remain largely unknown. Using an HCC mouse xenograft model and a human HCC cell line, in the present study we evaluated the efficacy of the ethanol extract of Livistona chinensis seed (EELC) against tumor growth in vivo and in vitro, and investigated the underlying molecular mechanisms.

\section{Materials and methods}

Materials and reagents. Dulbecco's modified Eagle's medium (DMEM), fetal bovine serum (FBS), penicillin-streptomycin, Trypsin-EDTA, TRIzol reagent, JC-1, caspase-3 and -9 colorimetric protease assay kits were purchased from Invitrogen (Carlsbad, CA, USA). SuperScript II reverse transcriptase was obtained from Promega (Madison, WI,USA). Bcl-2 and Bax antibodies, horseradish peroxidase (HRP)-conjugated secondary antibodies were obtained from Cell Signaling Technology (Beverly, MA, USA). The TUNEL assay kit was purchased from R\&D Systems (Minneapolis, MN, USA). A fluorescein isothiocyanate (FITC)-conjugated Annexin V apoptosis detection kit was obtained from Becton-Dickinson (San Jose, CA, USA). Tricin was provided by Professor Xinhua Lin from the Department of Pharmacology, Fujian Medical University. All other chemicals, unless otherwise stated, were obtained from Sigma-Aldrich (St. Louis, MO, USA).

Preparation of EELC. Livistona chinensis seeds (500 g) were extracted with $5,000 \mathrm{ml}$ of $85 \%$ ethanol using a refluxing method and were filtered. The resultant solution was concentrated to a relative density of 1.05 , and the dried powder of EELC was obtained by spraying desiccation method using a spray dryer (Buchi, Model B-290, Flawil, Switzerland). For animal experiments, the powder of EELC was dissolved in saline to a working concentration of $300 \mathrm{mg} / \mathrm{ml}$. The stock solution of EELC in cell-based experiments was prepared by dissolving EELC powder in 50\% DMSO to a stock concentration of $500 \mathrm{mg} / \mathrm{ml}$ and the working concentrations were made by diluting the stock solution in the cell culture medium. The final concentration of DMSO in the medium for all cell experiments was $<0.5 \%$.

HPLC-TOF/MS analysis. The samples were analyzed by HPLC-TOF/MS using a micrOTOF-Q spectrometer from Bruker Daltonics (Bremen, Germany) with an electrospray ionization (ESI) interface coupled with an HPLC Dionex UltiMate 3000 (Fig. 1). A Wonda Sil Herbal Medicine Column (150x4.6 mm; $5 \mu \mathrm{m}$, GL Sciences) was used for gradient sepa- ration. A linear gradient system consisted of mobile phase A ( $0.1 \%$ formic acid aqueous solution) and mobile phase $\mathrm{B}$ (acetonitrile containing $0.1 \%$ formic acid). The gradient elution profile was as follows: 0-5 $\mathrm{min}, 10 \% \mathrm{~B}$; $5-45 \mathrm{~min}, 10-95 \% \mathrm{~B}$; 45-50 $\mathrm{min}, 95 \% \mathrm{~B}$. The column was recycled with $10 \%$ solvent $\mathrm{B}$ for $10 \mathrm{~min}$, and equilibrated for another $2 \mathrm{~min}$ before using again. The column was maintained at $25^{\circ} \mathrm{C}$ with a flow rate of $0.4 \mathrm{ml} / \mathrm{min}$ during the gradient separation and column equilibration. The injection volume was $10 \mu \mathrm{l}$. Fig. $1 \mathrm{~A}$ and B shows HPLC profiles of EELC and a control sample tricin. The MS operating conditions were optimized as follows: the dry gas temperature was set at $180^{\circ} \mathrm{C}$, the flow rate was $3.0 \mathrm{l} /$ min, the nebulizer pressure was set at $2.0 \mathrm{bar}$, and the capillary voltage was at $-3.5 \mathrm{kV}$. Data were analyzed using Bruker Daltonics DataAnalysis 3.0 software.

Cell culture. Human HCC HepG2 cells were obtained from the American Type Culture Collection (ATCC, Manassas, VA, USA). The cells were grown in DMEM containing $10 \%(\mathrm{v} / \mathrm{v})$ FBS, and $100 \mathrm{U} / \mathrm{ml}$ penicillin and $100 \mu \mathrm{g} / \mathrm{ml}$ streptomycin in a $37^{\circ} \mathrm{C}$ humidified incubator with $5 \% \mathrm{CO}_{2}$. The cells were subcultured at $80-90 \%$ confluency.

Animals. Male BALB/c athymic (nude) mice (with an initial body weight of 20-22 g) were obtained from Shanghai SLAC Laboratory Animal Co., Ltd. (Shanghai, China) and housed under pathogen-free conditions with controlled temperature $\left(22^{\circ} \mathrm{C}\right)$, humidity, and a 12-h light/dark cycle. Food and water were provided ad libitum throughout the experiment. All animal treatments were performed strictly in accordance with the international ethical guidelines and the National Institutes of Health Guide concerning the Care and Use of Laboratory Animals. The experiments were approved by the Institutional Animal Care and Use Committee of Fujian University of Traditional Chinese Medicine.

In vivo nude mice xenograft study. HCC xenograft mice were produced with HepG 2 cells. The cells were grown in culture and then detached by trypsinization, washed, and resuspended in serum-free DMEM. Resuspended cells $\left(4 \times 10^{6}\right)$ mixed with Matrigel (1:1) were subcutaneously injected into the right flank of mice to initiate tumor growth. After 7 days of xenograft implantation when tumor size reached $\sim 3 \mathrm{~mm}$ in diameter, mice were randomized into two groups $(n=10)$ and intragastrically administered $3 \mathrm{~g} / \mathrm{kg}$ of EELC or saline daily, 5 days a week for 21 days. Body weight and tumor size were measured. Tumor size was determined by measuring the major (L) and minor (W) diameter with a caliper. The tumor volume was calculated according to the following formula: Tumor volume $=\pi / 6 \times \mathrm{L} \times \mathrm{W}^{2}$. At the end of the experiment, the animals were anesthetized with pelltobarbitalum natricum, and the tumor issue was removed and weighed. A portion of each tumor was fixed in $10 \%$ buffered formalin and the remaining tissue was snap-frozen in liquid nitrogen and stored at $-80^{\circ} \mathrm{C}$.

In situ apoptosis detection by TUNEL. The TUNEL reaction was carried out following treatment with EELC as previously described (32). The 4- $\mu \mathrm{m}$ sections of tumor samples were analyzed by TUNEL staining using TumorTACS In situ Apoptosis kit (R\&D Systems). Apoptotic cells were counted as 
A

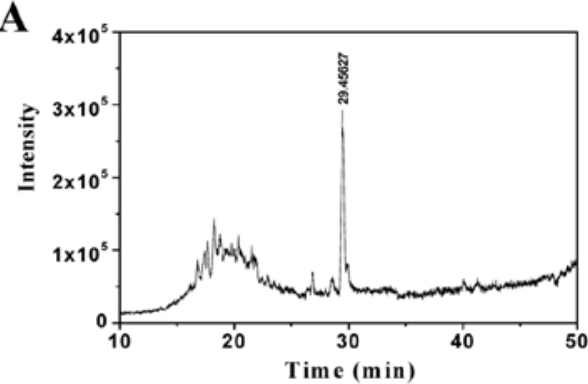

C

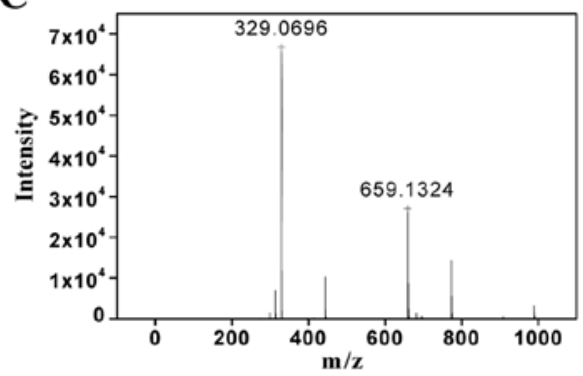

B

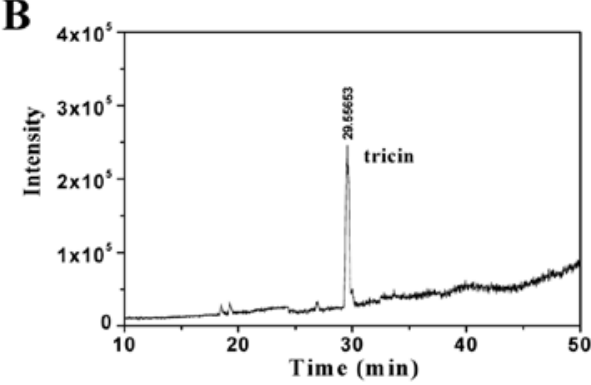

D

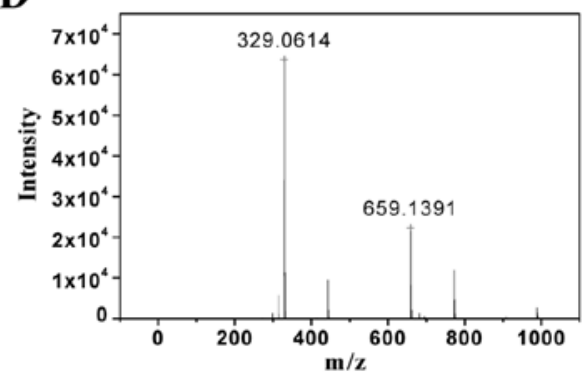

Figure 1. HPLC-TOF/MS analysis of EELC and tricin. HPLC profiles of (A) EESP and a (B) control sample. (C) Mass spectra of EELC and a (D) control sample. The control sample was tricin. The calculated MW of tricin was 329.0614.

DAB-positive cells (brown stained) at five arbitrarily selected microscopic fields at a magnification of $x 400$. TUNEL-positive cells were counted as a percentage of the total cells.

Evaluation of cell viability by MTT assay. Cell viability was assessed by the 3-(4,5-dimethylthiazol-2-yl)-2,5-diphenyltetrazolium bromide (MTT) colorimetric assay. HepG2 cells were seeded into 96 -well plates at a density of $1 \times 10^{4}$ cells/well in $0.1 \mathrm{ml}$ medium. The cells were treated with various concentrations of EELC for 6, 12 or $24 \mathrm{~h}$. Treatment with $0.5 \%$ DMSO was included as vehicle control. At the end of the treatment, $10 \mu \mathrm{l}$ MTT $(5 \mathrm{mg} / \mathrm{ml}$ in phosphate-buffered saline; PBS) were added to each well, and the samples were incubated for an additional $4 \mathrm{~h}$ at $37^{\circ} \mathrm{C}$. The purple-blue MTT formazan precipitate was dissolved in $100 \mu 1$ DMSO. The absorbance was measured at $570 \mathrm{~nm}$ using an ELISA reader (BioTek, Model ELx800, Winooski, Vermont, USA).

Detection of apoptosis by flow cytometry analysis with Annexin V/PI staining. Following incubation with various concentrations of EELC, apoptosis of HepG2 cells was determined by flow cytometry analysis using a fluorescenceactivated cell sorting (FACS) caliber (Becton-Dickinson) and Annexin V-fluorescein isothiocyanate (FITC)/Propidium iodide (PI) kit. Staining was performed according to the manufacturer's instructions. The percentage of cells in early apoptosis was calculated by Annexin V-positivity and PI-negativity, while the percentage of cells in late apoptosis was calculated by Annexin V-positivity and PI-positivity.

Measurement of mitochondrial membrane potential $(\Delta \Psi m)$ by flow cytometric analysis with $J C-1$ staining. JC-1 is a cationic dye that exhibits potential-dependent accumulation in mitochondria, indicated by a fluorescence emission shift from green to red, which can thus be used as an indicator of mitochondrial potential. In this experiment, $1 \times 10^{6}$ treated HepG2 cells were resuspended after trypsinization in $1 \mathrm{ml}$ of medium and incubated with $10 \mu \mathrm{g} / \mathrm{ml}$ of $\mathrm{JC}-1$ at $37^{\circ} \mathrm{C}, 5 \% \mathrm{CO}_{2}$, for $30 \mathrm{~min}$. Both red and green fluorescence emissions were analyzed by flow cytometry following JC-1 staining.

Analysis of caspase activation. The activities of caspase-3 and -9 were determined by a colorimetric assay using the caspase- 3 and -9 activation kits, following the manufacturer's instructions. Briefly, after treatment with various concentrations of EELC for 24 h, HepG 2 cells were lysed with the lysis buffer provided by the manufacturer for $30 \mathrm{~min}$ on ice. The lysed cells were centrifuged at $16,000 \times \mathrm{g}$ for $10 \mathrm{~min}$. The protein concentration of the clarified supernate was determined and $100 \mu \mathrm{g}$ of the protein were incubated with $50 \mu \mathrm{l}$ of the colorimetric tetrapeptides, Asp-Glu-Val-Asp (DEVD)-p-nitroaniline (pNA) (specific substrate of caspase-3) or Leu-Glu-His-Asp (LEHD)-pNA (specific substrate of caspase-9) at $37^{\circ} \mathrm{C}$ in the dark for $2 \mathrm{~h}$. Samples were read at $405 \mathrm{~nm}$ in an ELISA plate reader (BioTek, Model ELx800). The data were normalized to the activity of the caspases in control cells (treated with $0.5 \%$ DMSO vehicle) and represented as 'fold of control'.

RNA extraction and RT-PCR analysis. The expression of Bax and Bcl-2 genes were detected by RT-PCR as previously described (32). Briefly, total RNA from tumor tissues or HepG2 cells was isolated with TRIzol reagent. Oligo (dT)-primed RNA $(1 \mu \mathrm{g})$ was reverse-transcribed with SuperScript II reverse transcriptase (Promega) according to the manufacturer's instructions. The obtained cDNA was used to determine the mRNA amount of Bcl-2 or Bax by PCR. GAPDH was used as an internal control. The sequences of the primers used for amplification of Bcl-2, Bax, and GAPDH transcripts were: Bcl-2 forward, 5'-CAG CTG CAC CTG ACG CCC TT-3' and reverse, 5'-GCC TCC GTT ATC CTG GAT CC-3'; Bax forward, 
A

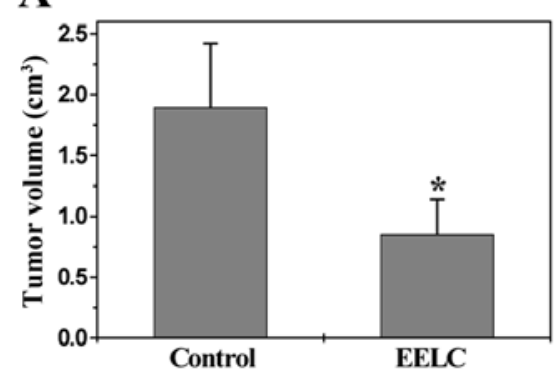

B

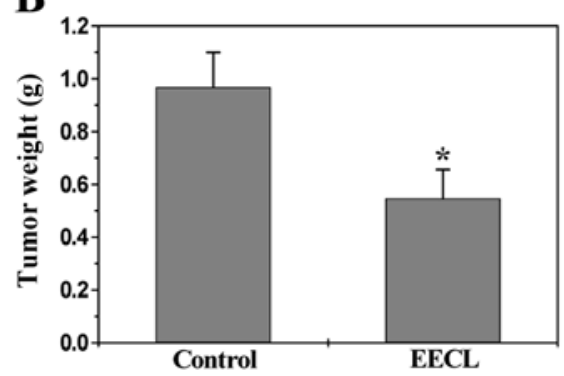

C

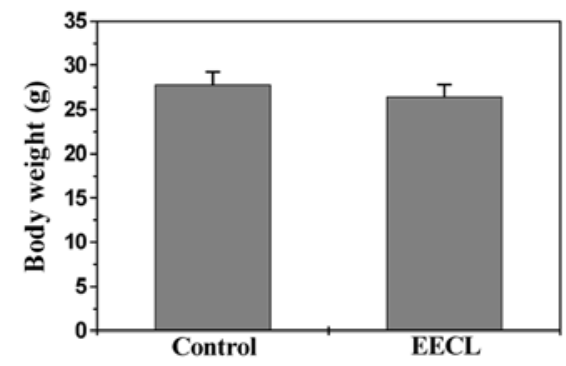

Figure 2. Effect of EELC on tumor growth in HCC xenograft nude mice. Following tumor development, the mice were given intragastrically administered $3 \mathrm{~g} / \mathrm{kg} / \mathrm{d}$ of EELC or saline daily, 5 days a week for 21 days. (A) Tumor volume, (B) tumor weight, and (C) body weight were measured at the end of the experiment. Data shown are averages with SD (error bars) from 10 individual mice in each group. ${ }^{*} \mathrm{P}<0.01$ vs. controls.

5'-TGC TTC AGG GTT TCA TCC AGG-3' and reverse, 5'-TGG CAA AGT AGA AAA GGG CGA-3'; GAPDH forward, 5'-GT CAT CCA TGA CAA CTT TGG-3' and reverse, 5'-GA GCT TGA CAA AGT GGT CGT-3'.

Immunohistochemistry. Immunohistochemical staining (IHS) for Bcl-2 and Bax was performed as previously described (33). Briefly, after fixing with $10 \%$ formaldehyde for $12 \mathrm{~h}$, tumor samples were processed conventionally for paraffin-embedded tumor slides. The slides were subjected to antigen retrieval and the endogenous peroxidase activity was quenched with hydrogen peroxide. After blocking non-specific proteins with normal serum in PBS (0.1\% Tween-20), slides were incubated with rabbit polyclonal antibodies against Bcl-2 and Bax (all in 1:200 dilution). After washing with PBS, slides were incubated with biotinylated secondary antibody followed by conjugated horseradish peroxidase (HRP)-labelled streptavidin (Dako), and then washed with PBS. The slides were then incubated with diamino-benzidine (DAB, Sigma) as the chromogen, followed by counterstaining with diluted Harris hematoxylin (Sigma). After staining, five high-power fields (x400) were randomly selected in each slide, and the average proportion of positive cells in each field was counted using the true color multi-functional cell image analysis management system (Image-Pro Plus, Media Cybernetics, USA). To rule out any non-specific staining, PBS was used to replace the primary antibody as a negative control.

Western blot analysis. HepG2 cells $\left(2 \times 10^{5}\right)$ were seeded into 6-well plates in $2 \mathrm{ml}$ medium and treated with various concentrations of EELC for $24 \mathrm{~h}$. Treated cells were lysed with mammalian cell lysis buffer containing protease and phosphatase inhibitor cocktails. The lysates were resolved in $12 \%$ SDS-PAGE gels and electroblotted. The PVDF membranes were blocked with $5 \%$ skimmed milk and probed with primary antibodies against Bcl-2, Bax or $\beta$-actin $(1: 1,000)$ overnight at $4^{\circ} \mathrm{C}$ and then with the appropriate HRP-conjugated secondary antibody followed by enhanced chemiluminescence detection.

Statistical analysis. All data are the means of three determinations. The data were analyzed using the SPSS package for Windows (Version 11.5). Statistical analysis of the data was performed with the Student's t-test and ANOVA. P $<0.05$ was considered to indicate statistically significant differences.

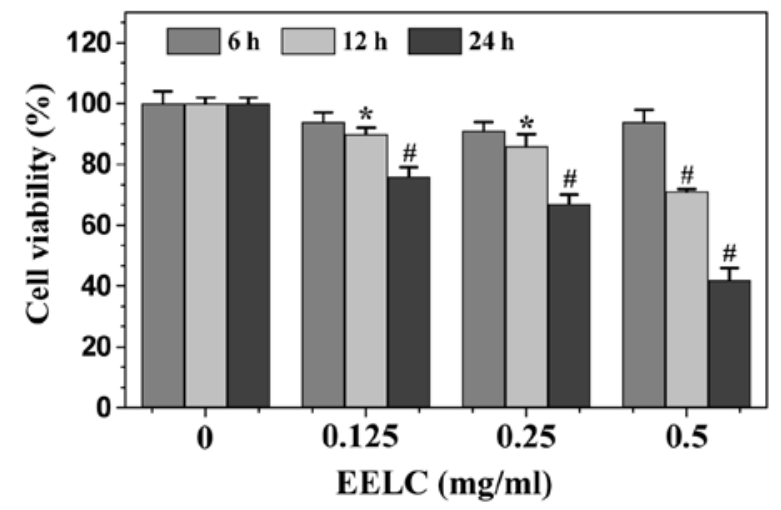

Figure 3. Effect of EELC on HepG2 cell viability. Cells were treated with various concentrations of EELC for the indicated time periods. Cell viability was determined by the MTT assay. The data were normalized to the viability of control cells ( $100 \%$, treated with $0.5 \%$ DMSO vehicle). Data are averages with SD (error bars) from at least three independent experiments. ${ }^{*} \mathrm{P}<0.05$, ${ }^{\#} \mathrm{P}<0.01$ vs. control cells.

\section{Results and Discussion}

EELC inhibits the growth of HCC in vivo and in vitro. The in vivo therapeutic efficacy of EELC against tumor growth was determined through comparison of tumor weight and volume in treated and control HCC xenograft mice, while its adverse effects were evaluated by measuring changes of body weight. As shown in Fig. 2A, EELC treatment resulted in a $50 \%$ decrease of tumor volume as compared to control (control, $1.90 \pm 0.52 \mathrm{~cm}^{3}$; EELC-treatment, $0.85 \pm 0.29 \mathrm{~cm}^{3}$; $\mathrm{P}<0.01$ ). Accordingly, the tumor weight per mouse in the EELC-treatment group was $43 \%$ less than that in the control group (control, $0.97 \pm 0.13 \mathrm{~g}$; EELC-treatment, $0.55 \pm 0.11 \mathrm{~g}$; $\mathrm{P}<0.01$ ) (Fig. 2B). However, EELC treatment had no effect on the changes of body weight (Fig. 2C). Taken together, it is suggested that EELC is potent in suppressing HCC growth in vivo, without apparent signs of toxicity. To evaluate the in vitro antitumor activity of EELC, we performed MTT assay to examine its effect on the viability of human HCC HepG2 cells. As shown in Fig. 3, treatment with 0, 0.125, 0.25 and $0.5 \mathrm{mg} / \mathrm{ml}$ of EELC for 6,12 or $24 \mathrm{~h}$, respectively, reduced cell viability by $6-24,9-33$ or $6-58 \%$, compared to untreated control cells $(\mathrm{P}<0.01)$, suggesting that EELC inhibits HCC cell growth in vitro in a dose- and a time-dependent manner. 
A

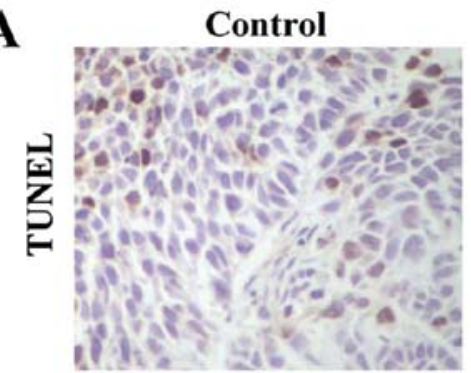

B

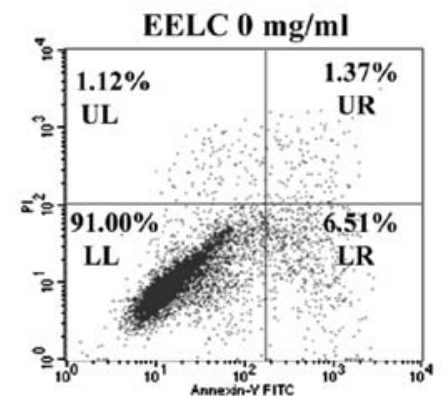

EELC $0.5 \mathrm{mg} / \mathrm{ml}$

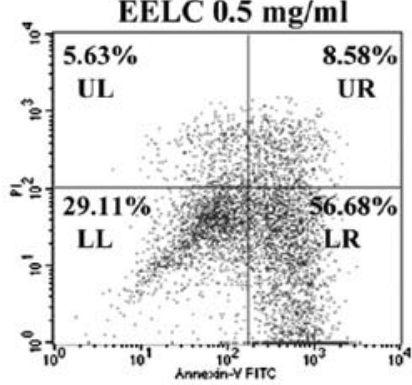

EELC
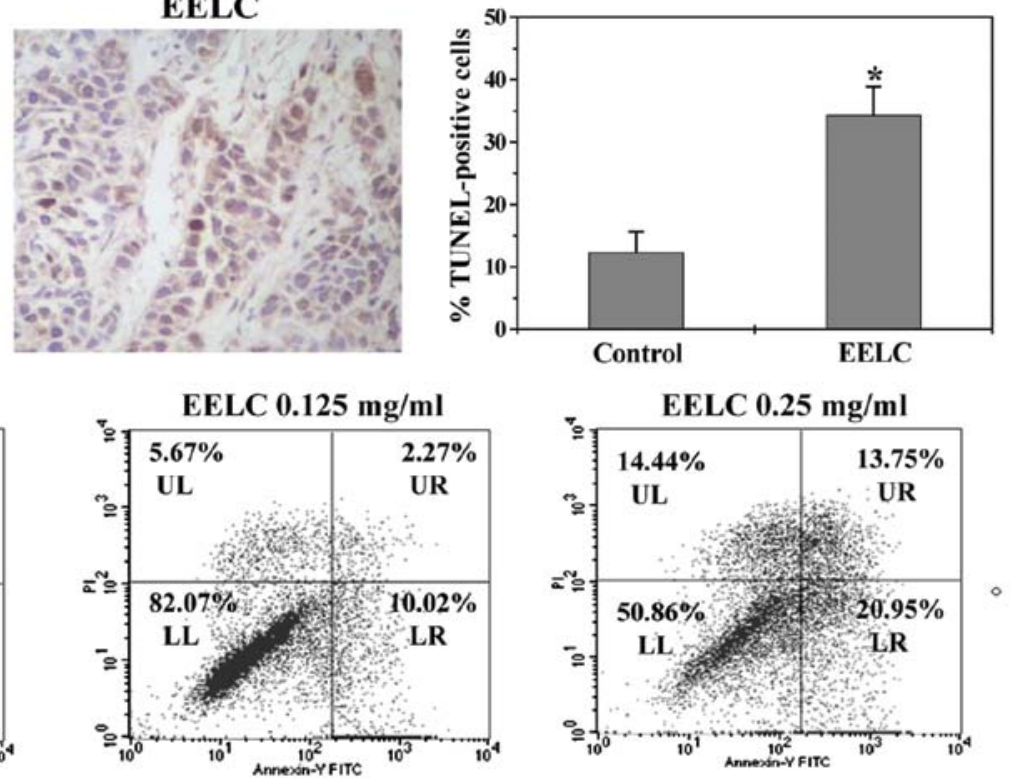

C

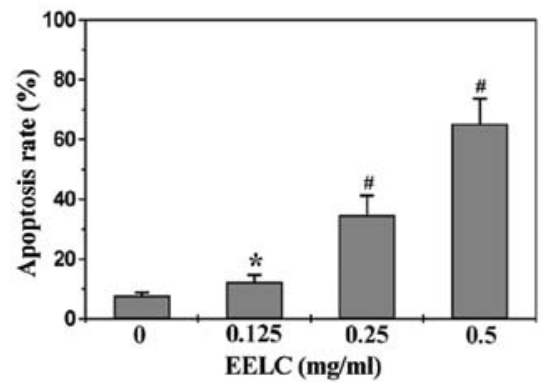

Figure 4. Effect of EELC on cell apoptosis in HCC xenograft mice and HepG2 cells. (A) At the end of the study, tumor tissues were processed for IHS for TUNEL. The representative images were captured at a magnification of $x 400$. Quantification of IHS assay was represented as percentage of positively-stained cells. Data shown are averages with SD (error bars) from 10 individual mice in each group. ${ }^{*} \mathrm{P}<0.01$ vs. controls. (B) Following treatment with the indicated concentrations of EELC for $24 \mathrm{~h}, \mathrm{HepG} 2$ cells were collected and stained with Annexin V/PI followed by FACS analysis. Images are representative of three independent experiments. (C) Quantification of FACS analysis. The data shown are averages with SD (error bars) from three independent experiments. "P<0.05, ${ }^{\text {"P}} \mathrm{P}<0.01$ vs. control cells.

EELC induces apoptosis in HCC xenograft tumor tissues and HepG2 cells. Cell apoptosis in tumors from HCC xenograft mice was evaluated via IHS for TUNEL. As shown in Fig. 4A, the percentage of TUNEL-positive cells was greater in tumors from EELC-treated mice as compared to controls (EELCtreatment, $34.33 \pm 4.52 \%$; control, $12.33 \pm 3.32 \%$; $\mathrm{P}<0.01$ ). Apoptosis of HepG2 cells was examined using Annexin V/PI staining followed by FACS analysis. In this assay, Annexin V/ PI double-negative population (labeled as LL in the FACS diagram) indicates viable cells, whereas Annexin V-positive/ PI-negative or Annexin V/PI double-positive population (labeled as LR or UR in the FACS diagram) represents cells undergoing early or late apoptosis, respectively. As shown in Fig. 4B and C, following treatment with $0,0.125,0.25$ and $0.5 \mathrm{mg} / \mathrm{ml}$ of EELC, the percentage of cells undergoing apoptosis (including the early and late apoptotic cells) was $7.8 \pm 1.05$, $12.3 \pm 2.34,34.7 \pm 6.53$ and $65.3 \pm 8.35 \%$, respectively $(\mathrm{P}<0.01$ or $0.05)$. These data demonstrate that EELC promotes HCC cell apoptosis both in vivo and in vitro.

EELC induces the loss of $\Delta \Psi_{m}$ in HepG2 cells. The mitochondrial-dependent pathway is the most common apoptotic pathway in vertebrate animal cells. Mitochondrial outer membrane permeabilization (MOMP), accompanied by the collapse of electrochemical gradient across the mitochondrial membrane, is a key commitment step in the induction of mitochondrial-dependent apoptosis, as it is the point of convergence for a large variety of intracellular apoptotic signaling pathways leading to the release of several apoptogenic proteins from the mitochondrial intermembrane space $(34,35)$. To investigate the mechanism of EELC's pro-apoptotic activity, we used FACS analysis with JC-1 staining to examine the change in $\Delta \Psi \mathrm{m}$ following EELC treatment. The membranepermeant JC-1 dye displays potential-dependent accumulation in mitochondria, indicated by a fluorescence emission shift from green $(\sim 525 \mathrm{~nm})$ to red $(\sim 590 \mathrm{~nm})$. Therefore, collapse of mitochondrial potential during apoptosis could be represented by a decrease in red fluorescence intensity. As shown in Fig. 5, upon treatment with EELC, the JC-1 fluorescence profile in HepG2 cells shifted from red-bright/green-bright signal to red-dim/green-bright pattern. The percentage of cells with reduced $\mathrm{JC}-1$ red fluorescence following treatment with $0,0.125,0.25$ and $0.5 \mathrm{mg} / \mathrm{ml}$ of EELC was $5.23 \pm 1.47$, $21.12 \pm 2.88,27.33 \pm 1.78$ and $33.57 \pm 5.73 \%$, respectively $(\mathrm{P}<0.01)$, suggesting that EELC dose-dependently induces the loss of $\Delta \Psi \mathrm{m}$ in HCC cells. 

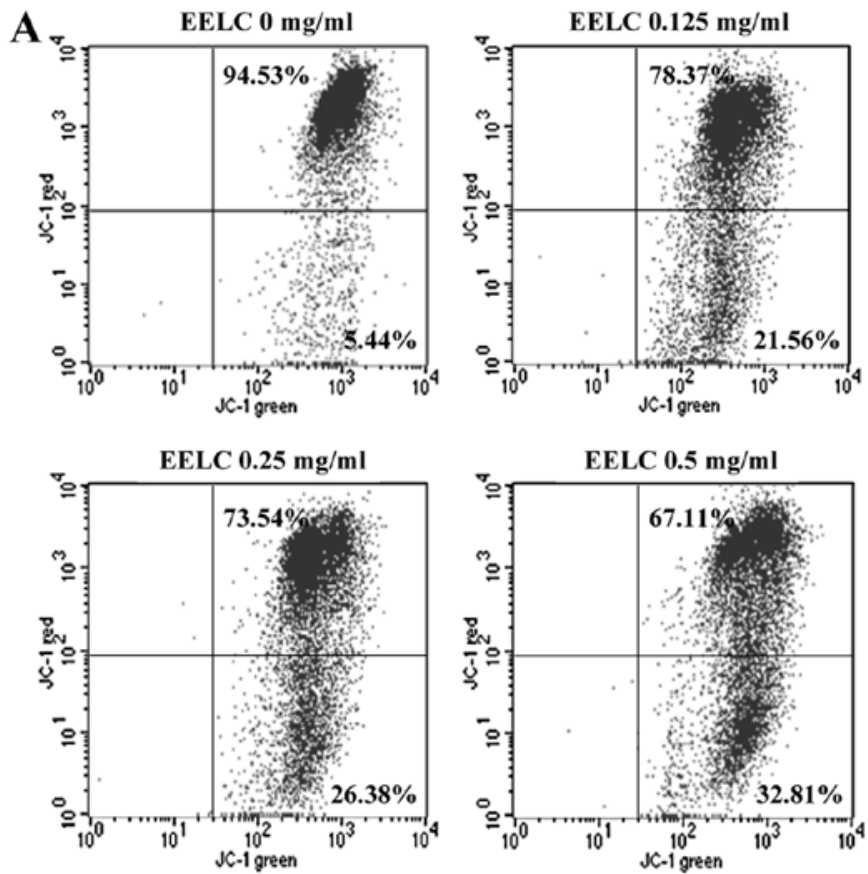

B

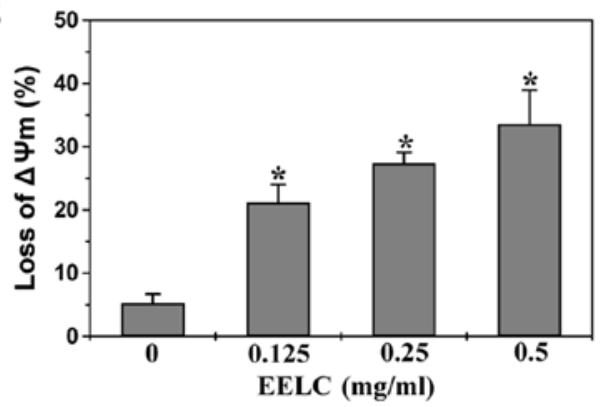

Figure 5. Effect of EELC on the loss of mitochondrial membrane potential in HepG2 cells. (A) Cells were treated with the indicated concentrations of EELC for $24 \mathrm{~h}$ and stained with JC-1. The mean JC-1 fluorescence intensity was detected using FACS analysis. Images are representative of three independent experiments. (B) Quantification of FACS analysis. Data shown are averages with SD (error bars) from three independent experiments. "P<0.01 vs. control cells.
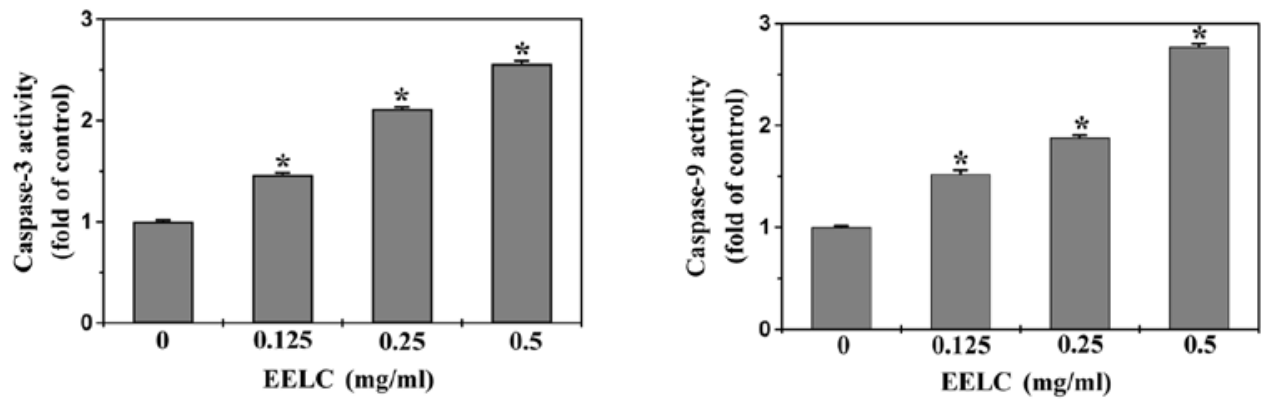

Figure 6. Effect of EELC on the activity of caspases in HepG2 cells. The cells were treated with the indicated concentrations of EELC for $24 \mathrm{~h}$. Caspase-3 and -9 activities were determined by a colorimetric assay. The data were normalized to the caspase activities within control cells (treated with $0.5 \%$ DMSO vehicle) and represented as 'fold of control'. Data are averages with SD (error bars) from at least three independent experiments. " $\mathrm{P}<0.05$ vs. control cells.

EELC induces the activation of caspase-9 and caspase- 3 in HepG2 cells. Caspases, represented by a family of cysteine proteases, are the key proteins that modulate the apoptotic response. Caspase-3, a key executioner of apoptosis, is activated by an initiator caspase such as caspase- 9 during mitochondrialmediated apoptosis. To identify the downstream effectors in the apoptotic signaling pathway, the activation of caspase-9 and caspase-3 was examined by a colorimetric assay using specific chromophores, DEVD-pNA (specific substrate of caspase-3) and LEHD-pNA (specific substrate of caspase-9). As shown in Fig. 6, EELC treatment significantly and dose-dependently induced activation of both caspase-9 and caspase-3 in HepG2 cells $(\mathrm{P}<0.05$, vs. untreated control cells).

EELC enhances the pro-apoptotic Bax/Bcl-2 ratio in $\mathrm{HCC}$ xenograft tumor tissues and HepG2 cells. Bcl-2 family proteins are key regulators of mitochondrial-mediated apoptosis, including anti-apoptotic members such as Bcl-2 and pro-apoptotic members such as Bax. MOMP is considered to occur through the formation of pores in the mitochondria by pro-apoptotic Bax-like proteins that can be inhibited by antiapoptotic Bcl-2-like members. Therefore, the ratio of active anti- and pro-apoptotic Bcl-2 family members is critical for determining the fate of cells. Higher Bcl-2 to Bax ratios are often found in various types of cancer (36), which not only confer a survival advantage to the cancer cells, but also cause resistance to chemo- and radio-therapies. To further study the mechanism of EELC's pro-apoptotic activity, we performed RT-PCR and IHS or western blotting to examine the mRNA and protein expression of $\mathrm{Bcl}-2$ and $\mathrm{Bax}$ in the $\mathrm{HCC}$ xenograft tumor tissues and the HepG2 cells. As shown in Figs. 7 and 8, EELC significantly reduced anti-apoptotic Bcl-2 mRNA levels both in the tumors of HCC mice and in the HepG2 cells, whereas the level of pro-apoptotic Bax mRNA was significantly increased following EELC treatment. The protein expression patterns of $\mathrm{Bcl}-2$ and Bax were similar to 
A

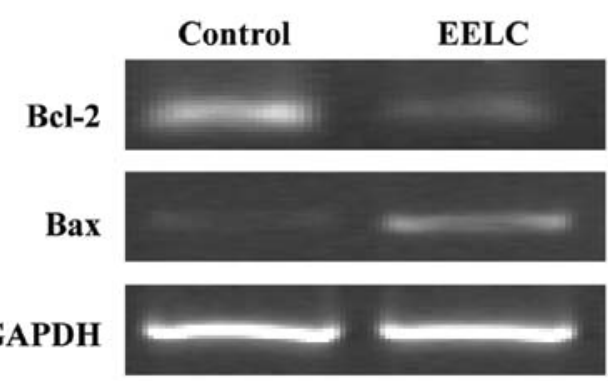

B

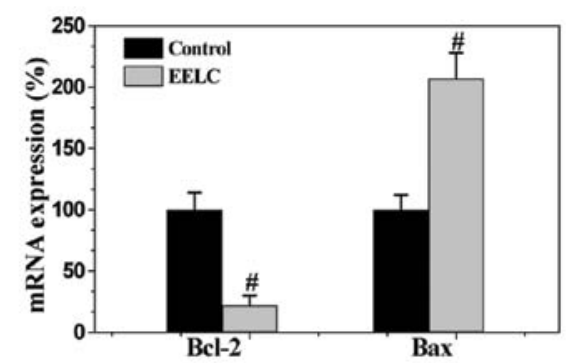

EELC

C

Control
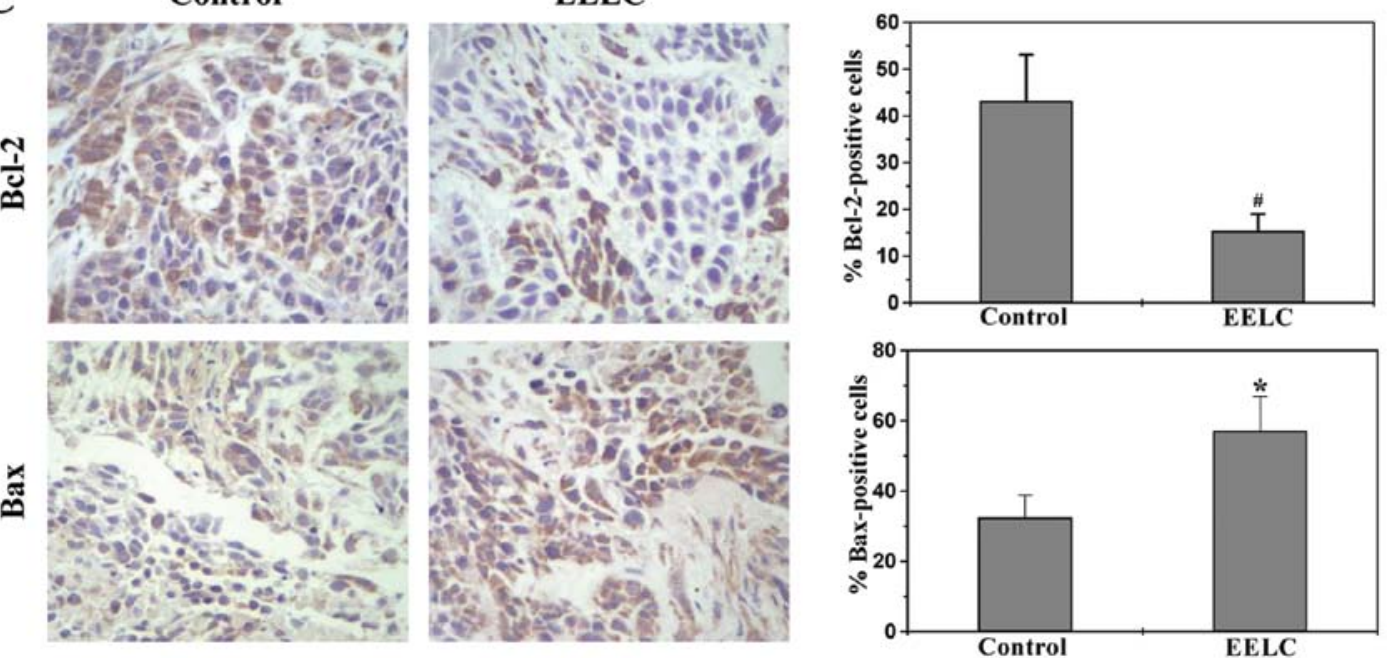

Figure 7. Effect of EELC on the expression of Bax and Bcl-2 in HCC xenograft mice. (A) The mRNA levels of Bcl-2 and Bax in tumor tissues were determined by RT-PCR. GAPDH was used as the internal control. Images are representative of 10 individual mice in each group. (B) Densitometric analysis. The data were normalized to the mean mRNA expression of untreated controls $(100 \%)$. ${ }^{*} \mathrm{P}<0.01$ vs. controls. (C) Tumor tissues were processed for IHS for Bcl-2 and Bax. The representative images were captured at a magnification of x400. Quantification of IHS assay was represented as percentage of positively-stained cells. Data shown are averages with SD (error bars) from 10 individual mice in each group. ${ }^{\mathrm{P}}<0.05,{ }^{\text {" }} \mathrm{P}<0.01$ vs. controls.

A

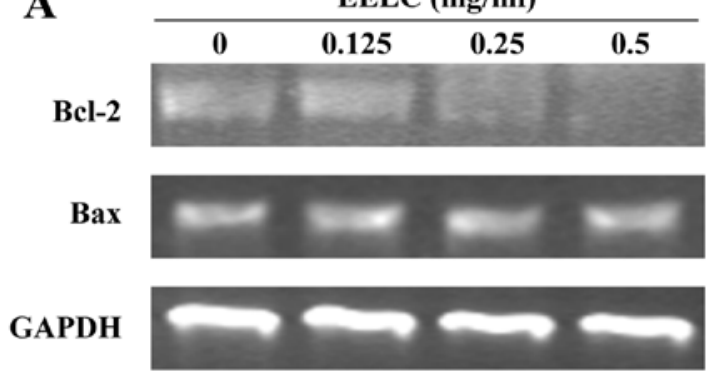

$\mathbf{B}$
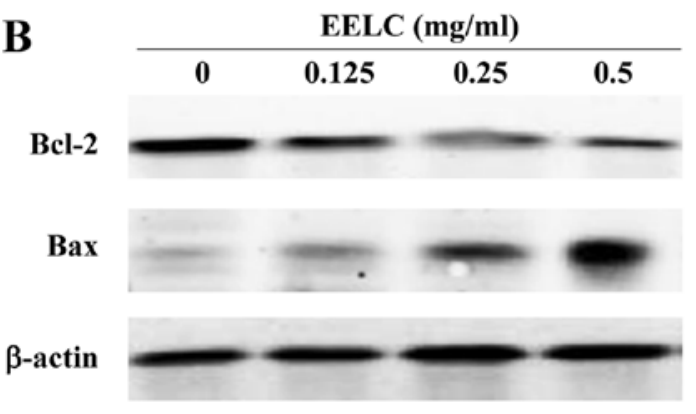

C

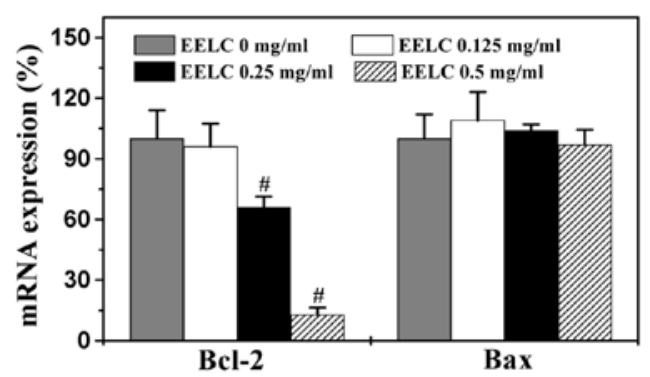

D

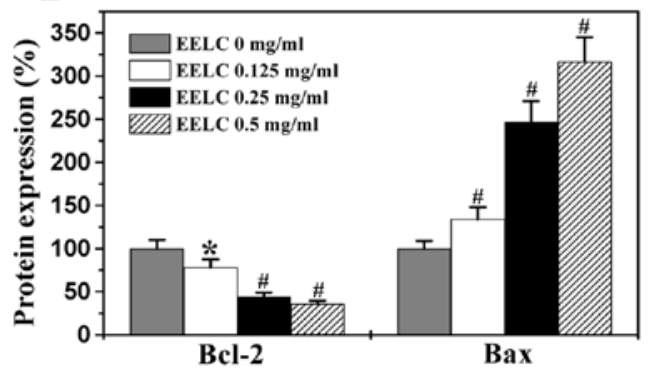

Figure 8. Effect of EELC on the expression of Bax and Bcl-2 in HepG2 cells. Cells were treated with the indicated concentrations of EELC for $24 \mathrm{~h}$. (A) The mRNA levels of Bax and Bcl-2 were determined by RT-PCR. (B) The protein expression levels of Bax and Bcl-2 were analyzed by western blotting. GAPDH and $\beta$-actin were used as the internal controls for the RT-PCR or western blotting assays, respectively. Data are representative of three independent experiments. (C and D) Densitometric analysis. The data were normalized to the mean mRNA (C) or protein (D) expression of untreated control cells (100\%), ${ }^{*} \mathrm{P}<0.05,{ }^{\#} \mathrm{P}<0.01$ vs. control cells. 
the patterns observed for the respective mRNA. Collectively, these data demonstrate that EELC promotes mitochondrialdependent apoptosis of $\mathrm{HCC}$ cells through upregulation in the pro-apoptotic $\mathrm{Bax} / \mathrm{Bcl}-2$ ratio.

In conclusion, herein we demonstrated for the first time that EELC inhibits HCC growth both in vivo and in vitro by promoting the mitochondrial-dependent apoptosis of cancer cells. Our findings suggest that the Livistona chinensis seed may be a potential novel therapeutic agent for the treatment of $\mathrm{HCC}$ and other types of cancer.

\section{Acknowledgements}

This study was sponsored by the National Natural Science Foundation of China (81073097 and 81202790).

\section{References}

1. Gomaa A, Khan S, Toledano M, Waked I and Taylor-Robinson S Hepatocellular carcinoma: epidemiology, risk factors and pathogenesis. World J Gastroenterol 14: 4300-4308, 2008.

2. Montalto G, Cervello M, Giannitrapani L, Dantona F, Terranova A and Castagnetta LA: Epidemiology, risk factors, and natural history of hepatocellular carcinoma. Ann NY Acad Sci 963: 13-20, 2002.

3. Sherman M: Hepatocellular carcinoma: epidemiology, risk factors, and screening. Semin Liver Dis 25: 143-154, 2005 .

4. Parkin D, Bray F, Ferlay J and Pisani P: Global cancer statistics, 2002. CA Cancer J Clin 55: 74-108, 2005.

5. Yeh C, Chen T, Chang M, Hsu C and Yeh T: Identification of NV-F virus DNA in hepatocellular carcinoma. J Med Virol 79: 92-96, 2007.

6. Levin B and Amos C: Therapy of unresectable hepatocellular carcinoma. N Engl J Med 332: 1294-1296, 1995.

7. Abou-Alfa G, Huitzil-Melendez F, O'Reilly E and Saltz L: Current management of advanced hepatocellular carcinoma. Gastrointest Cancer Res 2: 64-70, 2008.

8. Boose G and Stopper H: Genotoxicity of several clinically used topoisomerase II inhibitors. Toxicol Lett 116: 7-16, 2000.

9. Gordaliza M: Natural products as leads to anticancer drugs. Clin Transl Oncol 9: 767-776, 2007.

10. Newman D, Cragg G and Snader K: The influence of natural products upon drug discovery. Nat Prod Rep 17: 215-234, 2000.

11. Adams J and Cory S: The Bcl-2 apoptotic switch in cancer development and therapy. Oncogene 26: 1324-1337, 2007.

12. Cory S and Adams J: The Bcl-2 family: regulators of the cellular life-of-death switch. Nat Rev Cancer 2: 647-656, 2002.

13. Reed J: Mechanisms of apoptosis. Am J Pathol 157: 1415-1430, 2000.

14. Borner C: Bcl-2 family members: integrators of survival and death. Biochim Biophys Acta 1644: 71-72, 2004

15. Vaux D and Korsmeyer S: Cell death in development. Cell 96: 245-254, 1999

16. Gross A, McDonnell J and Korsmeyer S: Bcl-2 family members and the mitochondria in apoptosis. Genes Dev 13: 1899-1911, 1999.

17. Hsu Y, Wolter K and Youle R: Cytosol to membrane redistribution of members of the Bcl-2 family during apoptosis. Proc Natl Acad Sci USA 94: 3668-3672, 1997.

18. Wolter K, Hsu YT, Smith CL, Nechushtan A, Xi XG and Youle RJ: Movement of Bax from the cytosol to mitochondria. J Cell Biol 139: 1281-1292, 1997.
19. Tan C, Dlugosz PJ, Peng J, Zhang Z, Lapolla SM, Johnson AE, Andrews DW and Lin J: Auto-activation of the apoptosis protein Bax increases mitochondrial membrane permeability and is inhibited by Bcl-2. J Biol Chem 281: 14764-14775, 2006.

20. Antonsson B, Montessuit S, Lauper S, Eskes R and Martinou JC: Bax oligomerization is required for channel-forming activity in liposomes and to trigger cytochrome c release from mitochondria. Biochem J 345: 271-278, 2000.

21. Jürgensmeier JM, Xie Z, Deveraux Q, Ellerby L, Bredesen D and Reed JC: Bax directly induces release of cytochrome $\mathrm{c}$ from isolated mitochondria. Proc Natl Acad Sci USA 95: 4997-5002, 1998.

22. Kluck RM, Bossy-Wetzel E, Green DR and Newmeyer DD: The release of cytochrome $\mathrm{c}$ from mitochondria: a primary site for Bcl-2 regulation of apoptosis. Science 275: 1132-1136, 1997.

23. Yang J, Liu X, Bhalla K, Kim CN, Ibrado AM, Cai J, Peng TI and Jones DP: Prevention of apoptosis by Bcl-2: release of cytochrome c from mitochondria blocked. Science 275: 1129-1132, 1997.

24. Thomenius MJ, Wang NS, Reineks EZ, Wang $Z$ and Distelhorst CW: Bcl-2 on the endoplasmic reticulum regulates Bax activity by binding to $\mathrm{BH} 3$-only proteins. J Biol Chem 278: 6243-6250, 2003

25. Youle RJ and Strasser A: The BCL-2 protein family: opposing activities that mediate cell death. Nat Rev Mol Cell Biol 9: 47-59, 2008.

26. Yip KW and Reed JC: Bcl-2 family proteins and cancer. Oncogene 27: 6398-6406, 2008.

27. Zhao G, Dai S and Chen R: Dictionary of traditional Chinese medicine. Shanghai Scientific and Technical Publishers. Vol 2, pp2459-2460, 2006.

28. Cheueng S and Tai J: In vitro studies of the dry fruit of Chinese fan palm Livistona chinensis. Oncol Rep 5: 1331-1336, 2005.

29. Sartippour MR, Liu C and Shao ZM: Livistona extract inhibits angiogenesis and cancer growth. Oncol Rep 6: 1355-1357, 2001.

30. Wen $\mathrm{CH}, \mathrm{Rae} \mathrm{MH}$ and Lang MC: Selective downregulation of EGF receptor and downstream MAPK pathway in human cancer cell lines by active components partially purified from the seeds of Livistona chinensis R. Brown. Cancer Lett 248: 137-146, 2007.

31. Wang H, Li A, Dong XP and Xu XY: Screening of anti-tumor parts from the seeds of Livistona chinensis and its anti-angiogenesis effect. Zhong Yao Cai 315: 718-722, 2008 (In Chinese).

32. Cai Q, Lin J, Wei L, Zhang L, Wang L, Zhan Y, Zeng J, Xu W, Shen A, Hong Z and Peng J: Hedyotis diffusa willd inhibits colorectal cancer growth in vivo via inhibition of STAT3 signaling pathway. Int J Mol Sci 13: 6117-6128, 2012.

33. Wei L, Lin J, Xu W, Cai Q, Shen A, Hong Z and Peng J: Scutellaria barbata $\mathrm{D}$. don inhibits tumor angiogenesis via suppression of hedgehog pathway in a mouse model of colorectal cancer. Int J Mol Sci 13: 9419-9430, 2012.

34. Mantymaa P, Siitonen T, Guttorm T, Saily M, Kinnula V, Savolainen ER and Koistinen P: Induction of mitochondrial manganese superoxide dismutase confers resistance to apoptosis in acute myeloblastic leukaemia cells exposed to etoposide. Br J Haematol 108: 574-581, 2000.

35. Korper S, Nolte F, Rojewski MT, Thiel E and Schrezenmeier H: The $\mathrm{K}^{+}$channel openers diazoxide and NS1619 induce depolarization of mitochondria and have differential effects on cell $\mathrm{Ca}^{2+}$ in CD34+ cell line KG-1a. Exp Hematol 31: 815-823, 2003.

36. Kitada S, Pedersen IM,Schimmer AD and Reed JC: Dysregulation of apoptosis genes in hematopoietic malignancies. Oncogene 21: $3459-3474,2002$ 